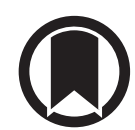

CrossMark

\title{
Targeting interleukin-13 in idiopathic pulmonary fibrosis: from promising path to dead end
}

\author{
Marlies S. Wijsenbeek ${ }^{1}$, Mirjam Kool (ib) and Vincent Cottin (10 ${ }^{2}$ \\ Affiliations: ${ }^{1}$ Dept of Pulmonary Medicine, Erasmus University Medical Center, Rotterdam, The Netherlands. \\ ${ }^{2}$ National Reference Center for Rare Pulmonary Disease, Louis Pradel Hospital; UMR754, Claude Bernard \\ University, Lyon, France.
}

Correspondence: Marlies S. Wijsenbeek, Dept of Pulmonary Medicine, Erasmus University Medical Center, Dr. Molenwaterplein 40, 3015 CA Rotterdam, The Netherlands. E-mail: m.wijsenbeek-lourensderasmusmc.nl

@ERSpublications

Targeting IL-4/IL-13 with SAR156597 failed to demonstrate an effect on lung function decline for patients with idiopathic pulmonary fibrosis; these results are in line with the negative results of two other trials targeting IL-13 in this disease http://ow.ly/J0HF30mAB3J

Cite this article as: Wijsenbeek MS, Kool M, Cottin V. Targeting interleukin-13 in idiopathic pulmonary fibrosis: from promising path to dead end. Eur Respir J 2018; 52: 1802111 [https://doi.org/10.1183/ 13993003.02111-2018].

The pathogenesis of idiopathic pulmonary fibrosis (IPF) is characterised by repeated subclinical injury to the alveolar epithelium, leading to injured alveoli, myofibroblast recruitment and activation, resulting in aberrant wound healing with uncontrolled matrix deposition and progressive fibrosis. However, inflammation and immunity are also thought to play a modulatory role in IPF pathogenesis. Data from IPF patients and experimental animal models have shown that type 2 inflammatory processes are activated in pulmonary fibrosis $[1,2]$. The main type 2 cytokines are interleukin (IL)-13 and IL-4, produced by T helper 2 (Th2) cells and type 2 innate lymphocytes; both are suggested to play a prominent role in fibrosis development $[1,2]$. As type 2 immunity is central in the immunopathology of allergic asthma, compounds targeting IL-13 and IL-4 have been developed for asthma [3-6].

\section{Trials targeting IL-13 in IPF}

Altogether, this prompted several stakeholders to further pursue blockage of IL-13 for the treatment of IPF. Two compounds (tralokinumab and lebrikikuzimab) developed for asthma were repurposed for IPF. Virtually at the same time, these two compounds targeting IL-13 and one newly developed compound (SAR156597) targeting both IL-13 and IL-4 were investigated in phase 2 randomised controlled trials (RCTs) in patients with IPF (figure 1).

In this issue of the European Respiratory Journal, RAGHU et al. [7] report on the results of the ESTAIR study, a phase 2 RCT evaluating the efficacy and safety of SAR156597, a bispecific monoclonal immunoglobulin G4 antibody binding and neutralising IL-4 and IL-13. Patients were randomly assigned 1:1:1 to placebo, or SAR156597 $200 \mathrm{mg}$ once every week or $200 \mathrm{mg}$ once every two weeks, for 52 weeks. Half of the patients were on background therapy with either nintedanib or pirfenidone. The study failed to demonstrate a favourable effect on the primary end-point, i.e. absolute change from baseline in per cent of predicted forced vital capacity (FVC) at 52 weeks, and had also no positive effect on secondary outcomes. The authors report numerically fewer acute exacerbations in the SAR156597 arms, although numbers of 


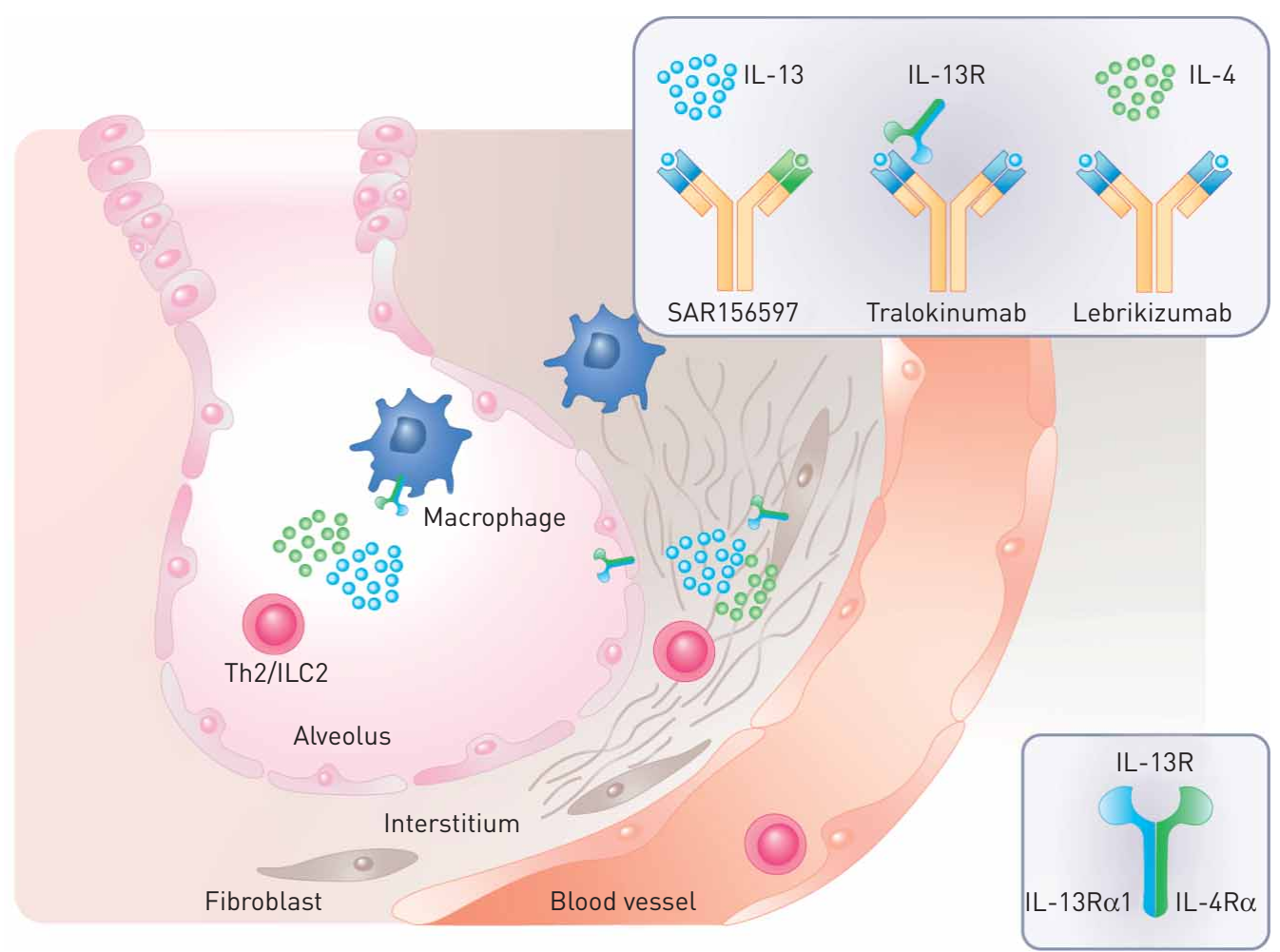

FIGURE 1 Schematic representation of an alveolus, interstitium and blood vessel. Expression of interleukin (IL)-13, IL-4 and IL-13 receptor (IL-13R) are depicted. In the top right corner, the three IL-13-targeting compounds and their specific mechanisms are depicted. Th2: T helper 2 cell; ILC2: type 2 innate lymphoid cell.

events were small. There was a significant decrease in the serum level of TARC (thymus and activation regulated chemokine) in patients treated with SAR156597 compared to placebo, confirming target engagement. In the weekly dosing arm, serious adverse events were more common than in the bi-weekly dosing arm.

The study should be seen in the light of the two other phase 2 RCTs targeting IL-13 (table 1). PArker et al. [8] reported on the safety and efficacy of tralokinumab 400 or $800 \mathrm{mg}$ every 4 weeks in treatment-naive patients with IPF. The primary end-point, the difference in absolute change from baseline in percent of predicted FVC at week 52, was not met (the study was prematurely stopped for futility). This study showed a high screen failure rate, and approval of nintedanib and pirfenidone during the study period slowed enrolment and likely contributed to a high drop-off rate. An accompanying editorial concluded that no definite conclusions could be drawn due to the limitations mentioned above [9]. In the two RIFF studies conducted in parallel $[10,11]$, patients with IPF were randomised to either lebrikizumab $250 \mathrm{mg}$ or placebo every 4 weeks, with or without background therapy with pirfenidone. None of the studies showed a treatment benefit on FVC change over 52 weeks. Some trends were observed on secondary end-points in one of the trials, which could, however, be a chance finding. Overall, four RCTs in IPF have failed to demonstrate a benefit of monoclonal antibodies targeting IL-13. Is this a dead end, or is it still worth pursuing the Th1/Th2 imbalance in IPF? Should we blame the choice of the target, the drugs, or the study design?

\section{What have we learned about targeting IL-13 from studies in asthma and IPF?}

In theory, SAR156597 therapy could be more promising than tralokinumab and lebrikizumab, as it neutralises both IL-13 and IL-4 (figure 1). Both IL-13 and IL-4 are elevated in IPF bronchoalveolar lavage fluid [12]. Whereas IL-13 promotes collagen production by lung fibroblasts [13], IL-4 can induce periostin production [14]. Periostin is elevated in IPF lungs and serum [15] and can promote fibrosis [16]. Next to reducing periostin, inhibition of IL-4 may also reduce the pool of Th2 cells [17] and possibly type 2 innate lymphocytes [18] in the airways, and may play a long-term role in reducing type 2 cytokine production.

In asthma, the only IL-13-targeting drug that has been thus far successful in two trials is dupilumab [3], which blocks both IL-13 and IL-4. The mechanism of action of dupilumab is different from that of 
TABLE 1 Key aspects of the four clinical trials evaluating interleukin (IL)-4/IL-13 antagonists in patients with idiopathic pulmonary fibrosis (IPF)

\begin{tabular}{|c|c|c|c|c|}
\hline & ESTAIR [7] & NCT01629667 [8] & RIFF A [10] & RIFF B [11] \\
\hline $\begin{array}{l}\text { Trial registration } \\
\text { number }\end{array}$ & NCT02345070 & NCT01629667 & NCT01872689 & NCT01872689 \\
\hline Compound & $\begin{array}{l}\text { SAR156597: humanised bispecific } \\
\text { IgG4 antibody binding and } \\
\text { neutralising IL-4 and IL-13 }\end{array}$ & $\begin{array}{l}\text { Tralokinumab: human } \lg G 4 \text { monoclonal } \\
\text { antibody neutralising IL-13 and } \\
\text { preventing receptor interaction }\end{array}$ & \multicolumn{2}{|c|}{$\begin{array}{l}\text { Lebrikizumab: humanised } \\
\text { monoclonal antibody that binds to } \\
\text { IL-13 }\end{array}$} \\
\hline Primary end-point & $\begin{array}{l}\text { Absolute change from baseline in } \\
\text { FVC \% pred at week } 52\end{array}$ & $\begin{array}{l}\text { Difference absolute change from } \\
\text { baseline in FVC \% pred at week } 52\end{array}$ & \multicolumn{2}{|c|}{$\begin{array}{l}\text { Annualised rate of decline in FVC \% } \\
\text { pred over } 52 \text { weeks }\end{array}$} \\
\hline $\begin{array}{l}\text { Randomised study } \\
\text { population }\end{array}$ & 109:108:108 per arm & 59:58:59 per arm & $76: 78$ per arm & 177:174 per arm \\
\hline $\begin{array}{l}\text { Result for primary } \\
\text { end-point }\end{array}$ & Not met & $\begin{array}{l}\text { Prematurely stopped for futility at an } \\
\text { interim analysis }\end{array}$ & Not met & Not met \\
\hline
\end{tabular}

SAR156597, as it binds to the IL-4R $\alpha$ chain, common to the IL-13 receptor (IL-13R) and the IL-4R, thereby preventing both IL-13 and IL-4 effector functions. In contrast, both tralokinumab and lebrikizumab were ineffective in several phase 3 trials in asthma [4-6].

These observations therefore favoured the use of a drug targeting both IL-4 and IL-13. However, in the current study [7], SAR156597 did not affect IPF progression in terms of FVC decline, suggesting that targeting the IL-13 pathway may not be clinically relevant in IPF. Interestingly, a positive trend was observed on acute exacerbations in SAR156597 treated patients, as reported in the pirfenidone plus lebrikizumab arm of the RIFF study [10,11]. Although the number of events was very small and these data should be interpreted cautiously, this raises the question about the potential role of type 2 driven processes in acute exacerbations.

\section{Challenges encountered in study design in the current IPF trial landscape}

The availability of two drugs (nintedanib and pirfenidone) that slow down disease progression has been an important step forward for patients with IPF $[19,20]$. However, this has significant implications for study design for potential novel compounds.

The current IPF trial landscape warrants a trial design with add-on to standard of care for ethical reasons and in order to avoid inclusion bias and recruitment failures. In such trials, the anticipated average decline in FVC for the whole patient group will be less than in a patient population without background therapy. Based on the placebo arms of previous trials, it is estimated that the average decline in FVC per year in patients without therapy is $\sim 200 \mathrm{~mL}$ per year, while in patients on either nintedanib of pirfenidone this decline is about halved [21]. This impacts power and sample size calculation using FVC as the primary outcome. In order to find a meaningful effect of a new compound on FVC, either sample size needs to be increased or the duration of the trial prolonged. In reality, it is rather complicated to take background disease modifying medications into account in trial design and analysis, as access to antifibrotics may vary by country, may change over time, and some patients discontinue treatments due to drug intolerance. 
Surveys estimated the overall uptake of antifibrotic drugs to be $50-60 \%$, with clear regional differences [22, 23]. In the study by RAGHU et al. [7], 51.1\% of patients were taking either nintedanib or pirfenidone, but the sample size calculation was not adjusted for background therapy, which may have led to an underpowering of the study. The differences in sample sizes of the studies targeting IL-13 are illustrative of the difference in assumptions that were likely made when calculating sample sizes and of the complex nature we are facing in current trial design. It is, however, unlikely that the results of the study may have been affected by sample size considerations.

There were some differences in inclusion criteria between the IL-13 IPF studies. On one side these differences may hamper detailed comparison or pooling of data, on the other hand broader and varying study populations will yield results that could be extrapolated to a wider patient population closer to everyday clinical practice. The SAR156597 study used the 2011 IPF diagnostic guidelines for inclusion [24], with the addition that patients with a possible usual interstitial pneumonia pattern on high-resolution computed tomography and additional evidence of traction bronchiectasis were also eligible. Together with broad pulmonary function criteria (FVC $\geqslant 40 \%$ pred and diffusing capacity of the lung for carbon monoxide $\geqslant 30 \%$ ), the inclusion criteria were even more lenient than in the INPULSIS trials, and probably one of the most pragmatic and close to real-life practice criteria used to date [19]. Nevertheless, screening failures occurred in $49 \%$ of cases (with the most common reason (36\%) being a positive interferon releasing test), consistent with other phase 2 and 3 trials in IPF. The impact of the new 2018 diagnostic guidelines [25] on trial eligibility is yet to be seen.

The fast-expanding field of RCTs with potential treatment options in IPF is very promising, but it also leads to competition for centres and patients. Careful trial design is needed with respect to sample size, study population and innovative end-points. Clinicians and patients invited to participate in early phase RCTs should be provided with compelling preclinical data supporting the use of new compounds to guide their choices. Ideally, collaboration between clinician, researchers, patients, and pharmaceutical companies should guide drug development, to best select promising pathways to explore, and to avoid crowds on the same paths - especially as some of them become dead ends.

Conflict of interest: M.S. Wijsenbeek reports grants and other from Boehringer Ingelheim, grants and other from Hoffman la Roche, other from Galapagos, outside the submitted work. All grants and fees were paid to M.S. Wijsenbeek's institution. M. Kool has nothing to disclose. V. Cottin reports personal fees for consultancy, lecture fees and support for travel to medical meetings from Actelion, grants, personal fees for consultancy, lecture fees and support for travel to medical meetings from Boehringer Ingelheim and Roche, personal fees for consultancy from Bayer/MSD, GSK and Galapagos, personal fees for adjudication committee membership from Gilead, personal fees for consultancy and lecturing from Novartis, grants from Sanofi, and personal fees for data and safety monitoring board work from Promedior and Celgene, outside the submitted work.

\section{References}

1 Zhu Z, Homer RJ, Wang Z, et al. Pulmonary expression of interleukin-13 causes inflammation, mucus hypersecretion, subepithelial fibrosis, physiologic abnormalities, and eotaxin production. J Clin Invest 1999; 103: 779-788.

2 Kolodsick JE, Toews GB, Jakubzick CV, et al. Protection from fluorescein isothiocyanate-induced fibrosis in IL-13-deficient, but not IL-4-deficient, mice results from impaired collagen synthesis by fibroblasts. J Immunol 2004; 172: 4068-4076.

3 Castro M, Corren J, Pavord ID, et al. Dupilumab efficacy and safety in moderate-to-severe uncontrolled asthma. N Engl J Med 2018; 378: 2486-2496.

4 Russell RJ, Chachi L, FitzGerald JM, et al. Effect of tralokinumab, an interleukin-13 neutralising monoclonal antibody, on eosinophilic airway inflammation in uncontrolled moderate-to-severe asthma (MESOS): a multicentre, double-blind, randomised, placebo-controlled phase 2 trial. Lancet Respir Med 2018; 6: 499-510.

5 Panettieri RA, Sjöbring U, Péterffy A, et al. Tralokinumab for severe, uncontrolled asthma (STRATOS 1 and STRATOS 2): two randomised, double-blind, placebo-controlled, phase 3 clinical trials. Lancet Respir Med 2018; 6 : 511-525.

6 Hanania NA, Korenblat P, Chapman KR, et al. Efficacy and safety of lebrikizumab in patients with uncontrolled asthma (LAVOLTA I and LAVOLTA II): replicate, phase 3, randomised, double-blind, placebo-controlled trials. Lancet Respir Med 2016; 4: 781-796.

7 Raghu G, Richeldi L, Crestani B, et al. SAR156597 in idiopathic pulmonary fibrosis: a phase 2, placebo-controlled study (DRI11772). Eur Respir J 2018; 52: 1801130.

8 Parker JM, Glaspole IN, Lancaster LH, et al. A phase 2 randomized controlled study of tralokinumab in subjects with idiopathic pulmonary fibrosis. Am J Respir Crit Care Med 2018; 197: 94-103.

9 Jones MG, Sgalla G, Richeldi L. Do randomized clinical trials always provide certain results? The case of tralokinumab in idiopathic pulmonary fibrosis. Am J Respir Crit Care Med 2018; 197: 9-10.

10 Swigris JJ, Ogura T, Scholand MB, et al. The RIFF study (cohort A): a phase II, randomized, double-blind, placebo-controlled trial of lebrikizumab as monotherapy in patients with idiopathic pulmonary fibrosis. Am J Respir Crit Care Med 2018; 197: A6167.

11 Maher TM, Kondoh Y, Corte TJ, et al. The RIFF study (cohort B): a phase II, randomized, double-blind, placebo-controlled trial of lebrikizumab in combination with pirfenidone in patients with idiopathic pulmonary fibrosis. Am J Respir Crit Care Med 2018; 197: A6168. 
12 Park S-W, Ahn M-H, Jang HK, et al. Interleukin-13 and its receptors in idiopathic interstitial pneumonia: clinical implications for lung function. J Korean Med Sci 2009; 24: 614-620.

13 Firszt R, Francisco D, Church TD, et al. Interleukin-13 induces collagen type-1 expression through matrix metalloproteinase- 2 and transforming growth factor- $\beta 1$ in airway fibroblasts in asthma. Eur Respir J 2014; 43: 464-473.

14 Takayama G, Arima K, Kanaji T, et al. Periostin: a novel component of subepithelial fibrosis of bronchial asthma downstream of IL-4 and IL-13 signals. J Allergy Clin Immunol 2006; 118: 98-104.

15 Okamoto M, Hoshino T, Kitasato Y, et al. Periostin, a matrix protein, is a novel biomarker for idiopathic interstitial pneumonias. Eur Respir J 2011; 37: 1119-1127.

16 Naik PK, Bozyk PD, Bentley JK, et al. Periostin promotes fibrosis and predicts progression in patients with idiopathic pulmonary fibrosis. Am J Physiol Lung Cell Mol Physiol 2012; 303: L1046-L1056.

17 Wurster AL, Withers DJ, Uchida T, et al. Stat6 and IRS-2 cooperate in interleukin 4 (IL-4)-induced proliferation and differentiation but are dispensable for IL-4-dependent rescue from apoptosis. Mol Cell Biol 2002; 22: 117-126.

18 Motomura Y, Morita H, Moro K, et al. Basophil-derived interleukin-4 controls the function of natural helper cells, a member of ILC2s, in lung inflammation. Immunity 2014; 40: 758-771.

19 Richeldi L, Bois du RM, Raghu G, et al. Efficacy and safety of nintedanib in idiopathic pulmonary fibrosis. $N$ Engl J Med 2014; 370: 2071-2082.

20 King TE, Bradford WZ, Castro-Bernardini S, et al. A phase 3 trial of pirfenidone in patients with idiopathic pulmonary fibrosis. N Engl J Med 2014; 370: 2083-2092.

21 Raghu G. Idiopathic pulmonary fibrosis: lessons from clinical trials over the past 25 years. Eur Respir J 2017; 50: 1701209 .

22 Wijsenbeek MS, Floricel F, Thonnard J, et al. Idiopathic pulmonary fibrosis - a worldwide review of "real" life' practice: experience from a treatment feasibility review in 41 countries. Eur Respir J 2018; 52: Suppl. 62, OA263.

23 Maher TM, Swigris JJ, Kreuter M, et al. Identifying barriers to idiopathic pulmonary fibrosis treatment: a survey of patient and physician views. Respiration 2018; 96: 1-11.

24 Raghu G, Collard HR, Egan JJ, et al. An official ATS/ERS/JRS/ALAT statement: idiopathic pulmonary fibrosis: evidence-based guidelines for diagnosis and management. Am J Respir Crit Care Med 2011; 183: 788-824.

25 Raghu G, Remy-Jardin M, Myers JL, et al. Diagnosis of Idiopathic Pulmonary Fibrosis. An Official ATS/ERS/JRS/ ALAT Clinical Practice Guideline. Am J Respir Crit Care Med 2018; 198: e44-e68. 